\title{
General duality for abelian-group-valued statistical-mechanics models
}

\author{
Sergio Caracciolo ${ }^{[1]}$, Andrea Sportiello ${ }^{[2]}$ \\ [1] Università degli Studi di Milano - Dip. di Fisica and INFN, \\ via Celoria 16, I-20133 Milano, and NEST-INFM, Italy \\ Mail address: Sergio.Caracciolo@mi.infn.it \\ [2] Scuola Normale Superiore and INFN - Sez. di Pisa, \\ Piazza dei Cavalieri 7, I-56100 Pisa, Italy \\ Mail address: Andrea.Sportiello@sns.it
}

\begin{abstract}
We introduce a general class of statistical-mechanics models, taking values in an abelian group, which includes examples of both spin and gauge models, both ordered and disordered.

The model is described by a set of "variables" and a set of "interactions". Each interaction is associated with a linear combination of variables; these are summarized in a matrix $J$. A Gibbs factor is associated to each variable (onebody term) and to each interaction.

Then we introduce a duality transformation for systems in this class. The duality exchanges the abelian group with its dual, the Gibbs factors with their Fourier transforms, and the interactions with the variables. High (low) couplings in the interaction terms are mapped into low (high) couplings in the one-body terms. If the matrix $J$ is interpreted as a vector representation of a matroid, duality exchanges the matroid with its dual.

We discuss some physical examples. The main idea is to generalize known models up to eventually include randomness into the pattern of interaction. We introduce and study a random Gaussian Model, a random Potts-like model, and a random variant of discrete scalar QED.

Although the classical procedure 'a la Kramers and Wannier does not extend in a natural way to such a wider class of systems, our weaker procedure applies to these models, too. We shortly describe the consequence of duality for each example.
\end{abstract}

PACS:05.50.+q, 64.60.Cn, 75.10.-b

keywords: Duality, Matroid Theory, Potts model.

\section{Introduction of the problem}

In statistical mechanics a notion of duality appeared first as a relation between the high- and low-temperature expansions in the two-dimensional Ising model [1]. In both expansions the partition function can be expressed as a sum on a gas of closed polymers: in the low-temperature picture, these polymers are the boundaries of the ferromagnetic domains, so we have an expansion in the deviations from the ordered regime; on the contrary, in the high-temperature picture the polymers are the terms of a Fourier expansion which expresses the deviation from a disordered regime of total decorrelation. So, in a certain sense, the duality relates energetic degrees of freedom to entropic degrees of freedom. Moreover, under the assumption of the existence of only one critical value for the temperature, it was possible to locate the phase-transition point before the exact solution of the model by Onsager. 
More formally, a duality transformation can be defined directly on the partition function of the model by means of two independent duality transformations, that is:

- The variables are located on the $k$-cells of a $D$ dimensional cell-complex (e.g. the 0-cells, or sites, for a spin model, the 1-cells, or bonds, for a gauge model), more precisely the physical state is a $k$-cochain taking values in an abelian group. The interactions are located on the $(k+1)$-cells (respectively the bonds and the plaquettes in the previous examples). The "algebraic" duality exchanges variables and interactions, and the dual physical state is a $(k+1)$-chain on the dual of the original group.

- The new variables have a gauge redundancy, and must obey a set of constraints: in other words, they must be a closed $(k+1)$-chain. The solution of the constraints leads to the "integration" of the $(k+1)$-chain to a $(k+2)$-chain. After this, the cellcomplex on which the model is defined is transformed into its dual counterpart. This "geometrical" duality of cellular complexes pictorially exchanges $k$-cells with $(D-k)$-cells, and the physical state is now a $(D-k-2)$-cochain.

This is the general strategy, explained in detail, for example, in the review part of [2], or in $[3$.

In this paper we wish to clarify the first ("algebraic") part of the duality transformation, by putting it in a more general context. We consider a rather general class of statistical-mechanical models in which the variables take values in an abelian group; each interaction term is associated to a particular linear combination of variables, but need not arise from any particular geometrical entity (such as a bond or a plaquette). By relaxing the requirement for a cell-complex structure, we can deal with a wider class of statistical models. We think that this more general notion of duality can be fruitful in the study of disordered systems.

This notion of duality is motivated, in fact, by the clauses-variables duality that we observed in a previous investigation in the zero-temperature behaviour of a model of random satisfiability [4], which has the structure of a disordered diluted interaction. The paper is organized as follows:

- In section 2 we introduce some mathematical formalism, in particular about the notion of duality and Fourier transform for generic abelian groups.

- In the sections from 3 to 8 we discuss our duality under general aspects: in section 3 we describe the most general setting for the duality; in section 4 we specialize the model to a formulation which is manifestly suitable for statistical mechanics models, and in section 5 we rederive the duality in this frame; in section 6 we show how to reconduct an extension of the original definition to the primitive formulation of the problem, which is reminiscent of a set of gauge fields coupled to the interaction terms; in section 7 we describe a particular extra-feature appearing in the model when delta constraints appears; finally in section 8 we introduce a sufficient criterium for self-duality.

- In sections from 9 to 13 we show the application of these general ideas to some concrete physical examples: first, in sections 9 and 10, a sort of random gaussian model, and a variant with random constraints; further, in sections 11 and 12 , a generalization of Potts model extended to random structures of interactions, and a discussion on how to recover, inside our framework, the traditional results for regular lattices; finally, in section 13, we discuss a concrete example of the general setting of section 6 , which is reminiscent of a scalar field theory coupled to electro-magnetism, both in the case of regular lattices and of disordered systems. 
- In section 14 we outline some conclusions, and introduce the perspective for a further generalization of the procedure, using different mathematical structures instead of an abelian group structure and the Fourier transform.

\section{Dual for an abelian group, Fourier transform and group homomorphisms}

In this section we introduce the Fourier transform for locally compact abelian groups, by means of the theory of characters, following the textbook of Kirillov and Gvishiani [5].

First, we introduce the concept of dual group. Let $G$ be a locally compact abelian group (we always write our abelian groups additively). Let $\mathcal{R}_{G}$ be the set of irreducible representations of $G$. It is well known that the irreducible representations of $G$ are all one-dimensional: thus, for each $\rho \in \mathcal{R}_{G}$, the representation matrix $U_{\rho}(g)$, is onedimensional and coincides with the character $\chi_{\rho}(g)$. The set $\mathcal{R}_{G}$ is itself an abelian group under ordinary composition (multiplication); we write this group additively and call it the dual group $\widehat{G}$. It is understood in the following that latin letters $g, h \ldots$ are elements of the original group $G$, and greek letters like $\rho, \tau \ldots$ are elements of the dual group $\widehat{G}$. If we adopt the notation

$$
\chi_{\rho}(g)=U_{\rho}(g)=e^{i \vartheta_{G}(\rho, g)},
$$

the following bilinear relations hold:

$$
\begin{aligned}
& \vartheta_{G}(\rho+\tau, g)=\vartheta_{G}(\rho, g)+\vartheta_{G}(\tau, g) ; \\
& \vartheta_{G}(\rho, g+h)=\vartheta_{G}(\rho, g)+\vartheta_{G}(\rho, h) .
\end{aligned}
$$

It can be shown that the dual group of $\widehat{G}$, denoted $\widehat{G}$, is naturally isomorphic to $G$.

In a consistent choice of notations, the characters of the dual group are derivable from the antisymmetric relation

$$
\vartheta_{G}(\rho, g)=-\vartheta_{\widehat{G}}(g, \rho),
$$

which is equivalent to

$$
\chi_{g}(\rho)=\overline{\chi_{\rho}(g)} .
$$

Given a function $f$ from $G$ to the complex plane, its Fourier transform $\widehat{f}$ is a function from the dual group $\widehat{G}$ to the complex plane, defined by

$$
\begin{array}{ll}
f: G \rightarrow \mathbb{C} & f(g)=\int_{\rho}^{*} e^{i \vartheta_{\widehat{G}}(g, \rho)} \widehat{f}(\rho) \\
\widehat{f}: \widehat{G} \rightarrow \mathbb{C} & \widehat{f}(\rho)=\int_{g} e^{i \vartheta_{G}(\rho, g)} f(g) .
\end{array}
$$

where $\int_{g}$ and $\int_{\rho}^{*}$ stand for a properly normalized Haar measure on $G$ and $\widehat{G}$ (see below). Subscripts on the bilinear forms $\vartheta_{G}$ and $\vartheta_{\widehat{G}}$ will be omitted when clear.

We introduce a notion of invariant $\delta$-function compatible with the measure introduced above:

$$
\int_{\rho}^{*} \overline{\chi_{\rho}\left(g^{\prime}\right)} \chi_{\rho}(g)=\delta_{G}\left(g^{\prime}-g\right) ; \quad \int_{g} \overline{\chi_{\rho^{\prime}}(g)} \chi_{\rho}(g)=\delta_{\widehat{G}}\left(\rho^{\prime}-\rho\right) ;
$$




\begin{tabular}{|c|c|c|c|}
\hline & $\left(\mathbb{Z}_{q} ; \mathbb{Z}_{q}\right)$ & $(U(1) ; \mathbb{Z})$ & $(\mathbb{R} ; \mathbb{R})$ \\
\hline $\int_{g}$ & $\frac{1}{q} \sum_{x=0}^{q-1}$ & $\int_{0}^{2 \pi} \frac{\mathrm{d} \theta}{2 \pi}$ & $\int_{-\infty}^{\infty} \mathrm{d} x$ \\
\hline $\int_{\rho}^{*}$ & $\sum_{\xi=0}^{q-1}$ & $\sum_{n=-\infty}^{+\infty}$ & $\int_{-\infty}^{\infty} \mathrm{d} \xi$ \\
\hline$\vartheta(g, \rho)$ & $2 \pi x \xi / q$ & $\theta n$ & $2 \pi x \xi$ \\
\hline$\delta_{G}(g)$ & $q \delta_{\mathrm{Kr}}(x)$ & $2 \pi \delta_{\text {Dirac }}(\theta)$ & $\delta_{\text {Dirac }}(x)$ \\
\hline$\delta_{\widehat{G}}(\rho)$ & $\delta_{\mathrm{Kr}}(\xi)$ & $\delta_{\mathrm{Kr}}(n)$ & $\delta_{\text {Dirac }}(\xi)$ \\
\hline
\end{tabular}

Table 1. Convention for the normalization of the Haar measure adopted in this paper for various pairs $(G, \widehat{G})$ of abelian groups. It is understood that for the direct product of groups the Haar measure is simply the product measure of the original ones.

which have the natural property

$$
\int_{g} f(g) \delta_{G}\left(g^{\prime}-g\right)=f\left(g^{\prime}\right) ; \quad \quad \int_{\rho}^{*} \widehat{f}(\rho) \delta_{\widehat{G}}\left(\rho^{\prime}-\rho\right)=\widehat{f}\left(\rho^{\prime}\right) .
$$

Inside the wide class of "physically natural" groups with the properties of being locally compact and compactly generated, the most general abelian group is the product of $\mathbb{R}, U(1), \mathbb{Z}$ and $\mathbb{Z}_{q}$ groups, so the suggested choice of normalizations of Table 1 covers all the possible situations. $\ddagger$

A homomorphism $\varphi$ from an abelian group $G_{1}$ to another abelian group $G_{2}$ is a map which preserves the group operation $\varphi\left(x_{1}+G_{1} x_{2}\right)=\varphi\left(x_{1}\right)+G_{2} \varphi\left(x_{2}\right)$, and maps the identity to the identity, $\varphi(0)=0$. We denote by $\operatorname{Hom}\left(G_{1}, G_{2}\right)$ the set of all homomorphisms from $G_{1}$ to $G_{2}$. Note that a homomorphism must preserve additive inverses, i.e. $\varphi(-g)=-\varphi(g)$. This follows from $\varphi(g)+\varphi(-g)=\varphi(0)=0$.

As we have seen in the previous paragraphs, from theory of characters we can introduce the dual groups $\widehat{G}_{1}$ and $\widehat{G}_{2}$. Following Kirillov ([5], Part III, statement 631), it is indeed possible to introduce a notion of dual homomorphism $\varphi^{T} \in \operatorname{Hom}\left(\widehat{G}_{2}, \widehat{G}_{1}\right)$

$\ddagger$ This fact derives essentially from three general results [6]

- Every locally compact, compactly generated abelian group $G$ is topologically isomorphic with $\mathbb{R}^{a} \times \mathbb{Z}^{b} \times K$ for some nonnegative integers $a$ and $b$ and some compact abelian group $K$.

- Let $K$ be a compact abelian group and let $U$ be a neighborhood of the identity in $K$. There is a closed subgroup $H$ of $G$ such that $H \subset U$ and $K / H$ is topologically isomorphic with $U(1)^{c} \times F$, where $c$ is a nonnegative integer and $F$ is a finite abelian group.

- Let $F$ be a finite abelian group. Then it is isomorphic to the group direct product of cyclic groups of prime power order (Kronecker decomposition theorem). 
which completes the diagram

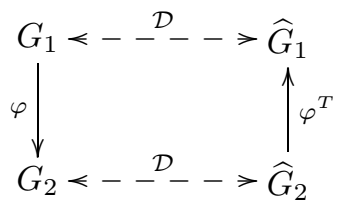

The dual homomorphism $\varphi^{T}$ acts according to the formula

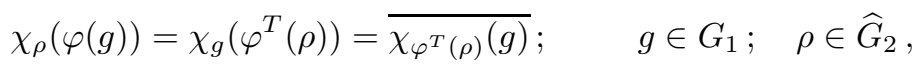

from which we derive

$$
\vartheta_{G_{2}}(\varphi(x), y)=\vartheta_{G_{1}}\left(\varphi^{T}(y), x\right) .
$$

It is easy to verify that $\varphi^{T}$ is a homomorphism from $\widehat{G}_{2}$ to $\widehat{G}_{1}$, as a consequence of the fact that $\vartheta(\rho, g)$ is a bilinear form $\S$.

\section{The general formulation of the duality}

In this section we wish to introduce a notion of duality for a wide class of systems in classical statistical mechanics.

In the most general form, given two abelian groups $G_{1}$ and $G_{2}$, we will consider the system defined by

- a function $F_{1}: G_{1} \rightarrow \mathbb{C}$;

- a function $\widehat{F}_{2}: \widehat{G}_{2} \rightarrow \mathbb{C}$;

- a homomorphism $\varphi \in \operatorname{Hom}\left(G_{1}, \widehat{G}_{2}\right)$;

and the Gibbs weight of a state $x \in G_{1}$ is given by

$$
\exp \left[-\mathcal{H}_{F_{1}, \widehat{F}_{2} ; \varphi}(x)\right]=F_{1}(x) \widehat{F}_{2}(\varphi(x)) .
$$

The partition function is defined as

$$
Z_{F_{1}, \widehat{F}_{2} ; \varphi}=\int_{x} F_{1}(x) \widehat{F}_{2}(\varphi(x)) .
$$

Applying Fourier transform to the function $\widehat{F}_{2}$ we obtain an algebraic relation:

$$
\begin{aligned}
& \int_{x} F_{1}(x) \widehat{F}_{2}(\varphi(x))=\int_{x} \int_{y} F_{1}(x) F_{2}(y) e^{i \vartheta_{G_{2}}(\varphi(x), y)} \\
& \quad=\int_{y} \int_{x} F_{2}(y) F_{1}(x) e^{i \vartheta_{G_{1}}\left(\varphi^{T}(y), x\right)}=\int_{y} F_{2}(y) \widehat{F}_{1}\left(\varphi^{T}(y)\right),
\end{aligned}
$$

from which we deduce that

$$
Z_{F_{1}, \widehat{F}_{2} ; \varphi}=Z_{F_{2}, \widehat{F}_{1} ; \varphi^{T}}
$$

Note that in the dual model the roles of the group $G_{1}$ and $G_{2}$ are interchanged, and the homomorphism $\varphi \in \operatorname{Hom}\left(G_{1}, \widehat{G}_{2}\right)$ is replaced by $\varphi^{T} \in \operatorname{Hom}\left(G_{2}, \widehat{G}_{1}\right)$.

$\S$ The homomorphism property descends from the bilinearity properties [2]:

$\chi_{\varphi^{T}\left(\rho_{1}+\rho_{2}\right)}(g)=\overline{\chi_{\rho_{1}+\rho_{2}}(\varphi(g))}=\overline{\chi_{\rho_{1}}(\varphi(g))} \cdot \overline{\chi_{\rho_{2}}(\varphi(g))}=\chi_{\varphi^{T}\left(\rho_{1}\right)}(g) \cdot \chi_{\varphi^{T}\left(\rho_{2}\right)}(g)=\chi_{\varphi^{T}\left(\rho_{1}\right)+\varphi^{T}\left(\rho_{2}\right)}(g)$. 


\section{Definition of a model with many degrees of freedom}

In this section we specialize the procedure described above to a more physical frame, in which extensive parameters appear both in the choice of the groups $G_{1}$ and $\widehat{G}_{2}$ and of the functions $F_{1}$ and $\widehat{F}_{2}$.

We introduce two extensive parameters: the first one, $r$, is called the number of variables, while the second one, $(n-r)$, is the number of interactions (they were called clauses in 4]).

Given a group $G$, we adopt $G_{1}=G^{\otimes r}$ and $\widehat{G}_{2}=G^{\otimes(n-r)}$. A state in $G_{1}$ is specified by a vector $x=\left(x_{1}, \ldots, x_{r}\right)$, and the homomorphism $\varphi$ is specified by a $r \times(n-r)$ matrix $J$, with elements $J_{i k} \in \operatorname{Hom}(G, G)$ acting on the left, such that $(x J)_{k}$ means $\sum_{i=1}^{r} J_{i k}\left(x_{i}\right)$. By convention we let the indices $k$ run from $r+1$ to $n$.

The functions $F_{1}$ and $\widehat{F}_{2}$ will be chosen to be factorized on the entries of the vectors:

$$
F_{1}[x]=\prod_{i=1}^{r} f_{i}\left(x_{i}\right) ; \quad \widehat{F}_{2}[y]=\prod_{k=r+1}^{n} f_{k}\left(y_{k}\right) .
$$

The weight functions $f_{1}, \ldots f_{r}$ are thus "one-body terms" (generalized magnetic fields), while the $f_{r+1}, \ldots f_{n}$ are "interaction terms".

Note that we have chosen the groups $G_{1}$ and $\widehat{G}_{2}$ to be direct products of the same group $G$. To avoid confusions in the application of the conventions of Table 1 . we specify that

$$
\begin{array}{rlrl}
\int_{[x] \in G_{1}} & \equiv \int_{x_{1}} \cdots \int_{x_{r}} ; & \vartheta_{G_{1}}\left(x^{\prime}, x\right)=\sum_{i} \vartheta_{G}\left(x_{i}^{\prime}, x_{i}\right) ; \\
\int_{[y] \in G_{2}}^{*} \equiv \int_{y_{r+1}}^{*} \cdots \int_{y_{n}}^{*} ; & \vartheta_{G_{2}}\left(y^{\prime}, y\right)=-\sum_{k} \vartheta_{G}\left(y_{k}, y_{k}^{\prime}\right) .
\end{array}
$$

We will sometime use a notation in which the two functions $F_{1}[x]$ and $\widehat{F}_{2}[x J]$ are "merged" into only one function $F[x B]=\prod_{j=1}^{n} f_{j}\left((x B)_{j}\right)$.

Denote with 1 the identity homomorphism $\varphi_{I} \in \operatorname{Hom}(G, G)$, such that $\varphi_{I}(x)=x$ for any $x$, and with 0 the null homomorphism $\varphi_{0} \in \operatorname{Hom}(G, G)$, such that $\varphi_{0}(x)=0$ for any $x$. The $r \times n$ matrix $B$ is thus defined as

$$
B_{i j}= \begin{cases}1 & i=j ; \quad 1 \leq j \leq r \\ 0 & i \neq j ; \quad 1 \leq j \leq r \\ J_{i j} & r+1 \leq j \leq n\end{cases}
$$

which can be written simply as

$$
B=\left[I_{r} \mid J\right]
$$

The invariance of the partition function under change of variables in the integration corresponds in terms of the matrix $B$ to the application of a special orthogonal transformation from the left (change of basis)

$$
Z[B]=\int_{G^{r}} F[x B] ; \quad Z[B]=Z[O B] \quad \forall O \in S O(r) .
$$


It results that, beyond the particular choice of the matrix $B$, a more fundamental notion of "pattern of interaction" can be established, which is independent from the choice of basis. This is the analogous of what happens in linear algebra, where the notion of linear independence is intrinsic with respect to the choice of basis. The introduction of this concept in mathematics dates up to the paper of Whitney [7, and is the foundation of a theory called Matroid Theory (cfr. [8] for a textbook). In particular, matroid is the name given to the intrinsic mathematical structure corresponding to our pattern of interaction, and a choice for the matrix $B$ is called a vectorial representation for the matroid. In the case in which the first $r \times r$ block of the matrix is the identity, we say that $B$ is a standard vectorial representation for the matroid. Note that our particular choice puts $B$ automatically in standard vectorial form.

In this frame, we can develop a physical intuition on the structure of our model. We could consider the $r$ entries of the vector $x$ as the variables of a statistical mechanic system. The first $r$ functions $f_{i}\left(x_{i}\right)$ collect the one-body contributions to the Gibbs factor, like one-body measure terms, or magnetic fields, or chemical potentials. The last $(n-r)$ functions $f_{k}\left((x J)_{k}\right)$ acts like $(n-r)$ terms of interaction, encoding informations like the strength of the couplings, or the nature of the clauses, etc.

The matrix $J$ describes how the variables interact. In the more general case, the entries of $J$ are homomorphisms from $G$ to itself. A specialization of this case is the one in which the entries of $J$ are in a commutative ring with unit, $\mathcal{R}$, and $G$ is an $\mathcal{R}$-module. A further specialization is the case in which $G$ is an arbitrary abelian group and $\mathcal{R}=\mathbb{Z}$. In fact, every additive group is an $\mathcal{R}$-module with $\mathbb{Z}$, where $n \cdot g=g+\ldots+g$ ( $n$ times $)$.

An example of this case is a scalar field theory in which the $r$ variables $\phi_{i}$ take a real value for each site $i$ of the lattice, and the interaction terms depend on $\left(\phi_{i}-\phi_{j}\right)^{2}$, where $(i, j)$ is a bond of the lattice. Another example is a Gauge Potts Model, in which the $r$ variables $s_{i}$ take a value in $\mathbb{Z}_{q}$ for each bond $i$ of the lattice, and the interaction terms depend on the linear combinations $\sum_{i \in \partial p} s_{i}$, where $p$ is a plaquette of the lattice. In fact in both these models all the coefficients of $J$ are in $\{0, \pm 1\}$. In the first case, the entry $J_{i j}$ is non-zero when the site labeled by $i$ is an extremum of the edge labeled by $j$, while in the second case this happens when the edge labeled by $i$ is on the border of the plaquette labeled by $j$, and the sign is determined by the choice of orientation for edges and plaquettes.

Nonetheless, other mathematical structures can be considered. A case which is not included in the situation above ( $G$ is an $\mathcal{R}$-module) is when the entries of $J$ are in a field $\mathbb{F}$, and $G$ is a finite-dimensional vector space over $\mathbb{F}\left(\right.$ say, $\mathbb{F}^{l}$ ). The field $\mathbb{F}$ could be, for example, the real field $\mathbb{R}$, the complex field $\mathbb{C}$, or a finite field $G F\left(q^{k}\right)$.

Moreover, we stress the fact that the generality of our formulation does not require any underlying geometrical structure for the system, and applies also to situations, typical of disordered mean-field models, in which such a structure does not exist.

\section{Duality for product groups}

Let us now repeat the procedure of the previous section specialized to our case, and write the corresponding duality transformation. The Boltzmann weight is

$$
\exp \left(-\mathcal{H}_{G ; f ; B}(x)\right)=\prod_{j=1}^{n} f_{j}\left((x B)_{j}\right) .
$$


The partition function is

$$
Z_{G ; f ; B}=\int_{[x]} \exp \left(-\mathcal{H}_{G ; f ; B}(x)\right)
$$

and, applying Fourier transform, can be restated as

$$
\begin{aligned}
Z_{G ; f ; B} & =\int_{[x]} \prod_{i=1}^{r} f_{i}\left(x_{i}\right) \prod_{k=r+1}^{n} f_{k}\left((x J)_{k}\right)=\int_{[x]} \int_{[y]}^{*} e^{i \vartheta_{G_{2}}(x J, y)} \prod_{i=1}^{r} f_{i}\left(x_{i}\right) \prod_{k=r+1}^{n} \widehat{f}_{k}\left(y_{k}\right) \\
& =\int_{[y]}^{*} \int_{[x]} e^{i \vartheta_{G_{1}}\left(-y J^{T}, x\right)} \prod_{k=r+1}^{n} \widehat{f}_{k}\left(y_{k}\right) \prod_{i=1}^{r} f_{i}\left(x_{i}\right)=\int_{[y]}^{*} \prod_{k=r+1}^{n} \widehat{f}_{k}\left(y_{k}\right) \prod_{i=1}^{r} \widehat{f}_{i}\left(\left(-y J^{T}\right)_{i}\right),
\end{aligned}
$$

from which we deduce that

$$
Z_{G ; f ; B}=Z_{\widehat{G} ; \widehat{f} ; \widehat{B}}
$$

where the dual $\widehat{B}$ of the matrix $B$ is intended exactly as in matroid theory (see [8], chapt. 2).

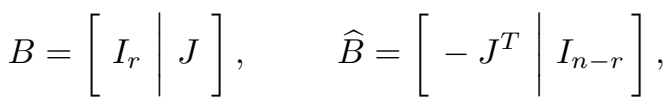

In the most general case of $J_{i j} \in \operatorname{Hom}(G, G)$, the quantities $\left(J^{T}\right)_{j i}$ are the dual homomorphisms $\widehat{J}_{i j}$ defined according to (6).

The duality interchanges the role of variables and interactions. Furthermore, if the functions $f_{j}$ are almost constant (low coupling, or high temperature), the functions $\widehat{f}_{j}$ are very peaked (high coupling, or low temperature), so the duality relates a regime of high (resp. low) temperature in the one-body terms with a regime of low (resp. high) temperature in the interaction terms, and vice versa.

\section{The coupling with gauge fields}

In this section we slightly generalize the formulation of the problem introduced in sections 3 and 4 and then we show that, with a change of variables, it can be recast in the original framework.

This analysis has one main reason: in section [13] we will describe the physical examples both of a scalar field theory, and of a discretized free electrodynamic theory, and show that our duality holds for both systems. Then we show that the duality holds also for the system in which electrodynamic is coupled to the scalar field in the traditional gauge-covariant way.

We want to show that this is a particular case of a general recipe, introduced in this section, and valid for the cases in which we introduce a set of gauge fields coupled to the terms of interaction. The change of variables corresponds to convert the basis of scalar fields and gauge fields into the basis of fields and covariant derivatives.

Given $2 k$ abelian groups $G_{1}, \ldots, G_{k}, G_{1}^{\prime}, \ldots, G_{k}^{\prime}$, we will consider the system defined by

- $k$ functions $f_{i}$ from the group $G_{i}$ to the field $\mathbb{C}$;

- $k$ functions $\widehat{g}_{i}$ from the group $\widehat{G}_{i}^{\prime}$ to the field $\mathbb{C}$;

- $2 k+1$ homomorphisms $\varphi_{i}$ between the groups $G_{i}$ and $\widehat{G}_{i}^{\prime}$ :

$$
0 \stackrel{\varphi_{-k}}{\longrightarrow} G_{k} \stackrel{\varphi_{-(k-1)}}{\longrightarrow} \cdots \stackrel{\varphi_{-1}}{\longrightarrow} G_{1} \stackrel{\varphi_{0}}{\longrightarrow} \widehat{G}_{1}^{\prime} \stackrel{\varphi_{1}}{\longrightarrow} \cdots \stackrel{\varphi_{k-1}}{\longrightarrow} \widehat{G}_{k}^{\prime} \stackrel{\varphi_{k}}{\longrightarrow} 0
$$


and the Gibbs weight of a state $x=\left(x_{1}, \ldots, x_{k}\right) \in \bigotimes G_{i}$ is

$$
\exp \left[-\mathcal{H}_{f, \widehat{g} ; \varphi_{i}}(x)\right]=\prod_{i=1}^{k} f_{i}\left(x_{i}-\varphi_{-i}\left(x_{i+1}\right)\right) \prod_{j=1}^{k} \widehat{g}_{j}\left(\varphi_{j-1} \cdots \varphi_{0}\left(x_{1}\right)\right) ;
$$

(it is understood that $x_{k+1}=0$ and $\varphi_{-k}\left(x_{k+1}\right)=0$ ).

Consider the change of variables $y_{i}=x_{i}-\varphi_{-i}\left(x_{i+1}\right)$, with Jacobian 1 . If we introduce the $k \times k$ matrix of homomorphisms

$$
\psi_{i j}=\varphi_{-(i-1)} \varphi_{-(i-2)} \cdots \varphi_{j-1},
$$

where the homomorphism $\psi_{i j}$ goes from $G_{i}$ to $\widehat{G}_{j}^{\prime}$, we could restate the Gibbs weight above as

$$
\exp \left[-\mathcal{H}_{f, \widehat{g} ; \varphi_{i}}(y)\right]=\prod f_{i}\left(y_{i}\right) \prod \widehat{g}_{j}\left(\sum_{l} \psi_{l j}\left(y_{l}\right)\right) .
$$

As a matrix of homomorphisms from the set of groups $\left\{G_{i}\right\}$ to the set of groups $\left\{\widehat{G}_{j}^{\prime}\right\}$ is a homomorphism between the product groups $\otimes G_{i}$ and $\otimes \widehat{G}_{j}^{\prime}$, we are in the conditions to apply of the general procedure we have outlined in section 3

The dual model interchanges the groups $G_{i}$ with the groups $G_{i}^{\prime}$, the functions $f_{i}$ with the functions $g_{i}$. As the transposed homomorphisms are given by

$$
\left(\psi^{T}\right)_{i j}=\left(\psi_{j i}\right)^{T}=\varphi_{i-1}^{T} \cdots \varphi_{-(j-1)}^{T},
$$

it corresponds to interchange the homomorphism $\varphi_{i}$ with the transposal homomorphism $\varphi_{-i}^{T}$. In this specialization of the general procedure, the relation (10) would read:

$$
Z_{f, \widehat{g} ;\left\{\varphi_{i}\right\}}=Z_{g, \widehat{f} ;\left\{\varphi_{-i}^{T}\right\}} \cdot
$$

In the case we deal with product groups, as described in section 4 the matrix $B$ will not be still in the standard form representation of Matroid Theory when expressed in the $x$ basis. Nevertheless, it will be in a clear block form with $k$ rows and $2 k$ columns of blocks. If we call $J_{i}$ the linear applications corresponding to the homomorphism $\varphi_{i}$, the block $B_{i j}$ would be given by

$$
B_{i j}= \begin{cases}I & i=j \leq k ; \\ -J_{-(k-i)} & i=j+1 \leq k ; \\ J_{0} J_{1} \cdots J_{j-k-1} & i=k ; j>k ; \\ 0 & \text { e.w. }\end{cases}
$$

The matrix $B$ can be easily diagonalized. This results in the change of basis from $x$ variables to $y$ variables in the sense described above. The resulting matrix $B^{\prime}$ will have blocks $B_{i j}^{\prime}$ given by

$$
B_{i j}^{\prime}= \begin{cases}I & i=j \leq k \\ 0 & i \neq j ; j \leq k \\ J_{-(i-1)} J_{-(i-2)} \cdots J_{j-k-1} & j>k\end{cases}
$$

Now that $B^{\prime}$ is in the standard form representation, we can apply duality, and then, inverting the diagonalization procedure described above, restate the dual problem in the original block form. The transformation interchanges the matrices $J_{i}$ with $J_{-i}^{T}$, with a minus sign on $J_{0}$, which goes into $-J_{0}^{T}$. 


\section{The solution of delta constraints}

A general feature of our duality in the factorized case is that the resolution of constraints taking the form of delta functions has a dual counterpart in the removal of trivial constant factors, and vice versa. This feature can be seen in a transparent way in a simple unstructured problem, shown in section 10 but it is more commonly used when the duality is combined with other geometric properties of the systems, as, for example, in the case of models defined over regular finite-dimensional cell-complexes. Such a situation will be described in section 12

Consider a system with $r$ variables and $n-r$ interactions, in the standard form described above, in the case in which the first $r$ functions $f_{i}(x)$ are constant. For simplicity, say that the last $(n-r)$ functions $f_{k}(y)$ are all equal to a certain $f(y)$. The model is described by the Gibbs weight

$$
\exp [-\mathcal{H}(x)]=\prod_{j=r+1}^{n} g\left((x J)_{j}\right) .
$$

Following the duality procedure, the dual Gibbs weight is given by

$$
\exp [-\widehat{\mathcal{H}}(\xi)]=\prod_{i=1}^{r} \delta\left(\left(-\xi J^{T}\right)_{i}\right) \prod_{j=r+1}^{n} \widehat{f}\left(\xi_{j}\right) .
$$

In the following, assume for simplicity that the incidence matrix $J$ has maximal rank. $\ddagger$ In this case it can be written in standard form $J=\left(I, J^{\prime}\right)$ by mean of a change of variables, and a relabeling of the last $(n-r)$ indices. So we can rewrite the Gibbs weights above as

$$
\begin{gathered}
\exp [-\mathcal{H}(x)]=\prod_{j=r+1}^{2 r} f\left(x_{j}\right) \prod_{j=2 r+1}^{n} f\left(\left(x J^{\prime}\right)_{j}\right) \\
\exp [-\widehat{\mathcal{H}}(\xi)]=\prod_{i=1}^{r} \delta\left(-\xi_{i}^{\mathrm{dep}}-\left(\xi^{\mathrm{ind}}{J^{\prime}}^{T}\right)_{i}\right) \prod_{j=r+1}^{2 r} \widehat{f}\left(\xi_{j}^{\mathrm{dep}}\right) \prod_{j=2 r+1}^{n} \widehat{f}\left(\xi_{j}^{\mathrm{ind}}\right) ;
\end{gathered}
$$

where in the last expression we already stressed that the variables $\xi^{\text {ind }}$ form an independent set, while the variables $\xi^{\text {dep }}$ can be expressed in terms of them when solving the delta constraints. In this case we obtain

$$
\exp [-\widehat{\mathcal{H}}(\xi)]=\prod_{j=r+1}^{2 r} \widehat{f}\left(\left(-\xi^{\text {ind }}{J^{\prime}}^{T}\right)_{j}\right) \prod_{j=2 r+1}^{n} \widehat{f}\left(\xi_{j}^{\text {ind }}\right) .
$$

This is exactly the Gibbs weight obtained applying duality directly to the weight in the (29), considered as a system with $r$ variables, labeled with the indices from $r+1$ to $2 r$, and $n-2 r$ interactions, labeled with the indices from $2 r+1$ to $n$.

$\ddagger$ Matrices of non-maximal rank $r^{\prime}<r$ would only imply trivial overall factors of the kind $|\mathbb{F}|^{\left(r-r^{\prime}\right)}$, or the necessity of fixing "by hands" $\left(r-r^{\prime}\right)$ variables, as for example in planar Potts model, where a factor $q$ originates from this fact, or in the XY-Model - Coulomb Gas relation, in which we should avoid to sum over the variable associated to one special site, in order to fix a "ground" for the potential. 
In conclusion, we draw a scheme which resumes the procedure described above:

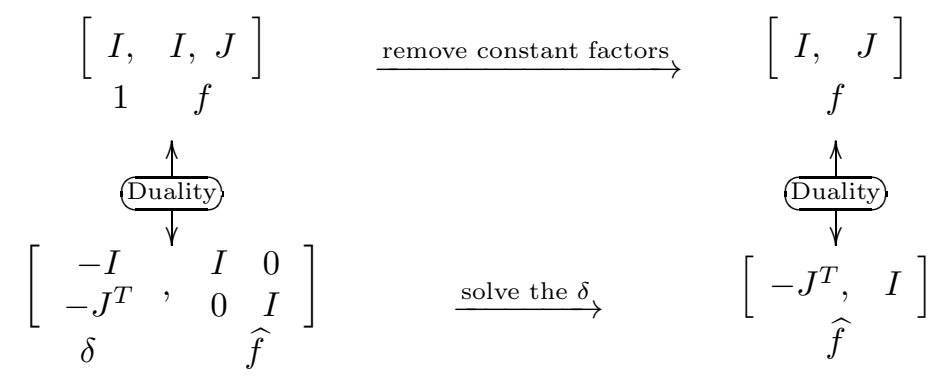

\section{A sufficient condition for self-duality}

Duality is particularly useful whenever it is possible to recover that a system, under suitable conditions, is self-dual.

In the following, let us denote by $X$ the instance of a problem (say, the lattice, the set of couplings,...), and by $\sigma$ a configuration of the system (say, the values of all the spins in the system).

Duality means that there is an involution $\{X\} \rightarrow\{X\}$ such that

$$
Z[\widehat{X}]=f[X] Z[X]
$$

with $f[X]$ an analytic function of $X$.

Of course a sufficient condition for an instance $X^{*}$ to be self-dual is that it is a fixed point of the involution

$$
\widehat{X_{*}}=X_{*} .
$$

If there is a group of invariances $\mathcal{U}$ such that

$$
\forall u \in \mathcal{U} \quad Z[u X]=Z[X]
$$

then a weaker condition for self-duality can be stated. It is sufficient that some $u \in \mathcal{U}$ exists such that

$$
u \widehat{X_{*}}=X_{*} .
$$

At this point, we make a short digression on disordered systems. For these systems the variable $X$ describing the instance is itself a random variable, distributed over some space $\{X\}$ with some normalized measure $\mu(X)$ given a priori. We are interested in the thermodynamic quantities (that is, thermalized over $\sigma$ ) of a system in a given instance $X$, furtherly averaged over the distribution of disorder $\mu(X)$. For example, the average free energy, up to $\beta$ factors, is given by

$$
F=\overline{\log Z}=\int \mathrm{d} \mu(X) \log Z[X] .
$$

Source variables can be introduced to calculate the other thermodynamic quantities. For example, to calculate the average magnetization we can introduce a magnetic field $h$, and obtain

$$
F(h)=\overline{\log Z(h)} ; \quad M=\left.\frac{\partial}{\partial h} F(h)\right|_{h=0}
$$


similarly, to calculate the average two-point function $\overline{\left\langle\sigma_{i} \sigma_{j}\right\rangle}$ we can introduce a vector of local magnetic fields $\left\{h_{i}\right\}$, one per site, and obtain

$$
\overline{\left\langle\sigma_{i} \sigma_{j}\right\rangle}=\int \mathrm{d} \mu(X)\left\langle\sigma_{i} \sigma_{j}\right\rangle_{X}=\left.\frac{\partial^{2}}{\partial h_{i} \partial h_{j}} F(h)\right|_{h=0}
$$

The action of duality on the space of instances $\{X\}$ naturally induces an action on the space of normalized measures over $\{X\}$

$$
\widehat{\mu}(\widehat{X})=\left|\frac{\partial X}{\partial \widehat{X}}\right| \mu(X(\widehat{X})) .
$$

The condition for self-duality now applies to measures. The trivial condition for a measure $\mu^{*}(X)$ to define a self-dual problem is

$$
\widehat{\mu^{*}}(X)=\mu^{*}(X) \text {. }
$$

Analogously to what we did above, if we have a group $\mathcal{U}$ of invariances for the problem, we can make this condition weaker. If we have a normalized Haar measure $\int_{\mathcal{U}}$ on the group, the condition which exploits the symmetries of the problem is

$$
\int_{\mathcal{U}} \widehat{\mu^{*}}(u X)=\int_{\mathcal{U}} \mu^{*}(u X) .
$$

Of course this condition can be fulfilled, by using the invariance of the Haar measure, if for any fixed $X$ there is an element of the group $\mathcal{U}$ such that

$$
\widehat{\mu^{*}}(u X)=\mu^{*}\left(u v_{X} X\right) .
$$

In practice, in the case of a disordered system, we have a measure on the space of $n$-uples of functions $\left\{f_{i}\right\}$, and of $r \times(n-r)$ incidence matrices $J$. In the case in which $r=n / 2$, all the first $r$ functions $f_{i}$ are equal to a certain $f(x)$ and all the last $r$ functions $f_{k}$ are equal to its Fourier transform $\widehat{f}(x)$, the dual set of function is identical to the original one, and we only need to check self-duality on the measure for the incidence matrix, $\mu(J)$.

Let the functions $f(x)$ and $\widehat{f}(x)$ be invariant under a subgroup of the automorphisms of the space $G, \mathcal{A} \subseteq \operatorname{Aut}(G)$.

The elements $u$ of the group of invariance $\mathcal{U}$ are such that $Z_{f ; J}=Z_{f ; u J}$.

They are of the form $u=\left(\pi ;\left\{a_{i}\right\},\left\{a_{k}\right\}\right)$ with $\pi \in \mathfrak{S}_{\{1, \ldots, r\}} \times \mathfrak{S}_{\{r+1, \ldots, n\}}$, where $\mathfrak{S}_{\Lambda}$ is the permutation group on the set $\Lambda$, and $a_{i}, a_{k}$ in $\mathcal{A}$, with $i=1, \ldots, r$ and $k=r+1, \ldots, n$. The action of $u$ on a matrix $J$ is

$$
(u J)_{i j}=a_{j} J_{\pi(i) \pi(j)} a_{i},
$$

from which we have that a consistent group composition is

$$
u^{\prime} u=\left(\pi^{\prime} \pi ;\left\{a_{\pi^{\prime}(i)} a_{i}^{\prime}\right\},\left\{a_{k}^{\prime} a_{\pi^{\prime}(k)}\right\}\right) .
$$

The condition for self-duality (36) above, in our case can be restated as the existence for each matrix $J$ of an element $u_{J} \in \mathcal{U}$ such that

$$
\mu^{*}\left(u_{J}\left(-J^{T}\right)\right)=\mu^{*}(J) .
$$

Note that the Jacobian of the transform $J \rightarrow-J^{T}$ is one. Exploiting the group $\mathcal{U}$ of invariances for the problem, we can restrict our attention to measures which are central functions for the group $\mathcal{U}$. If we introduce the two-element group generated by the transformation $\iota: J \rightarrow-J^{T}$, the self-dual measures are the (fewer) central functions on the (larger) group $\mathcal{U} \rtimes\{e, \iota\}$. 


\section{An easy example: the Gaussian model}

As a first easy application of formula (17) we will consider a simple model exactly solvable via Gaussian integration. Consider the system described by equation (15), specialized to the case in which $G=\mathbb{R}$ and the functions $\left\{f_{j}\right\}$ are

$$
\begin{aligned}
f_{i}(x) & =e^{-x^{2} / 2 \sigma_{i}^{2}} & & \text { for } \quad i=1, \ldots, r ; \\
f_{r+k}(x) & =e^{-x^{2} / 2 \tau_{k}^{2}} & & \text { for } \quad k=1, \ldots, n-r .
\end{aligned}
$$

We will introduce a notation ad hoc to label the partition function of this system:

$$
Z_{\text {Gauss }}\left(J ;\left\{\sigma_{i}\right\},\left\{\tau_{k}\right\}\right) \equiv Z_{\mathbb{R}, B,\left\{f_{j}\right\}},
$$

where the expressions (38) above for the $\left\{f_{j}\right\}$ and the vectorial representation $B=\left[I_{r} \mid J\right]$ are understood.

We could rewrite equation (15) in the more familiar form

$$
\exp \left(-\mathcal{H}_{G ; f ; B}(x)\right)=\prod_{j=1}^{n} f_{j}\left((x B)_{j}\right)=e^{-\frac{1}{2} x^{\dagger}\left(S^{-2}+J T^{-2} J^{T}\right) x},
$$

where $S_{i i^{\prime}}=\sigma_{i} \delta_{i i^{\prime}}$ and $T_{k k^{\prime}}=\tau_{k} \delta_{k k^{\prime}}$. Using the convention of Table 1 for the measure $\int_{x}$ in the case $X=\mathbb{R}$ (that is, writing down the proper number of ( $\left.2 \pi\right)$ factors), and applying the formula for Gaussian integration, we have for the partition function

$$
Z_{\text {Gauss }}\left(J ;\left\{\sigma_{i}\right\},\left\{\tau_{k}\right\}\right)=\int \mathrm{d} x e^{-\frac{1}{2} x^{\dagger}\left(S^{-2}+J T^{-2} J^{T}\right) x}=\frac{(2 \pi)^{\frac{r}{2}} \operatorname{det} S}{\sqrt{\operatorname{det}\left(I_{r}+M^{T} M\right)}},
$$

where we defined

$$
M=T^{-1} J^{T} S .
$$

From equation (17) we have that the dual partition function is

$$
Z_{\text {Gauss }}\left(-J^{T} ;\left\{\tau_{k}^{-1}\right\},\left\{\sigma_{i}^{-1}\right\}\right)=\frac{(2 \pi)^{\frac{n-r}{2}} \operatorname{det} T^{-1}}{(2 \pi)^{\frac{r}{2}} \operatorname{det} S} Z_{\text {Gauss }}\left(J ;\left\{\sigma_{i}\right\},\left\{\tau_{k}\right\}\right),
$$

while, applying the formula for Gaussian integration on the dual formulation,

$$
Z_{\text {Gauss }}\left(-J^{T} ;\left\{\tau_{k}^{-1}\right\},\left\{\sigma_{i}^{-1}\right\}\right)=\int \mathrm{d} \eta e^{-\frac{1}{2} \eta^{\dagger}\left(T^{2}+J S^{2} J^{T}\right) \eta}=\frac{(2 \pi)^{\frac{n-r}{2}} \operatorname{det} T^{-1}}{\sqrt{\operatorname{det}\left(I_{n-r}+M M^{T}\right)}} .
$$

So the consequences of the duality can be restated in the algebraic equation

$$
\operatorname{det}\left(I_{r}+M^{T} M\right)=\operatorname{det}\left(I_{n-r}+M M^{T}\right),
$$

which is indeed true, although not totally trivial. $\S$

$\S$ Consider the formal power series in $z$ :

$$
\begin{aligned}
& \ln \left(\operatorname{det}\left(I-z M^{T} M\right)\right)= \operatorname{tr}\left(\ln \left(I-z M^{T} M\right)\right)=-\sum_{n=1}^{+\infty} \frac{z^{n}}{n} \operatorname{tr}\left(M^{T} M\right)^{n} \\
&=-\sum_{n=1}^{+\infty} \frac{z^{n}}{n} \operatorname{tr}\left(M M^{T}\right)^{n}=\operatorname{tr}\left(\ln \left(I-z M M^{T}\right)\right)=\ln \left(\operatorname{det}\left(I-z M M^{T}\right)\right) .
\end{aligned}
$$




\section{A randomly constrained Gaussian Model}

In this section we introduce a model in which some Gaussian distributed variables in $\mathbb{R}^{D}$ are subjected to a set of random constraints.

This model is a first example in which the matrix of the homomorphisms $B$ really contains non-trivial group homomorphisms. Furthermore, it exhibits in a clear way the general feature of our duality described in section 7 the resolution of delta constraints has a dual counterpart in the removal of trivial constant factors.

The model is defined as follows. Let $x_{i}, i=1, \ldots, r$, be $D$-dimensional real vectors, and introduce the $(n-r)$ linear combinations

$$
y_{k}^{\alpha}=\sum_{i=1}^{r} \sum_{\beta=1}^{D} x_{i}^{\beta} J_{i k}^{\beta \alpha}
$$

with $k=r+1, \ldots, n$, defined by the matrices $J_{i k}$. We will consider the case $2 r>n$.

The Gibbs weight of a configuration is

$$
\mathrm{d} x \exp \left[-\mathcal{H}_{J}(x)\right]=\mathrm{d}^{D} x_{1} \cdots \mathrm{d}^{D} x_{r} \exp \left(-\frac{1}{2 \sigma^{2}} \sum_{i=1}^{r} x_{i}^{2}\right) \cdot \prod_{k=r+1}^{n} \delta\left(y_{k}, 0\right) .
$$

Working in the spirit of disordered systems, we would have a probability measure over the quenched disorder variables $J_{i k}$. Introducing the Haar measure $\mathrm{d} \mu_{D}$ over the group of special orthogonal transformations $S O(D)$, a possible choice is to take the variables $J_{i k}$ as i.i.d. variables, distributed with $\mathrm{d} \mu_{D}$ :

$$
\mathrm{d} \mu(J)=\prod_{\substack{i=1, \ldots, r \\ k=r+1, \ldots, n}} \mathrm{~d} \mu_{D}\left(J_{i k}\right) .
$$

The problem has a large gauge group, which allows for the reduction of the matrix $J$ in a standard vectorial form. We have mainly three class of manipulations on $B$ which do not modify the value of the partition function:

- permutations of the columns:

$$
J_{i k} \quad \longrightarrow \quad J_{i \sigma(k)} \quad \sigma \in \mathfrak{S}_{n-r}
$$

- global rotations on rows and columns:

$$
J_{i k}^{\beta \alpha} \quad \longrightarrow \quad R_{(i)}^{\beta \beta^{\prime}} J_{i k}^{\beta^{\prime} \alpha^{\prime}} R_{(k)}^{\alpha \alpha^{\prime}} \quad R_{(j)} \in S O(D), 1 \leq j \leq n ;
$$

- full Gauss algorithm on the rows:

$$
J_{i k} \quad \longrightarrow \quad C_{i j} J_{j k} \quad C \in S O(r) .
$$

Thus, by change of variables we can put the matrix $J$ in the form

$$
J^{\prime}=\left(\begin{array}{c}
Y \\
-I_{n-r}
\end{array}\right) \text {. }
$$

Remark that this can be done always when the rank of $J$ is maximal, and this happens with probability 1 for our measure $\mathrm{d} \mu(J)$. 
This procedure of diagonalization corresponds to the algebraic resolution of the delta functions. In fact, the linear combinations are

$$
\left(y^{\prime}\right)_{k}^{\alpha}=\sum_{i=1}^{r} \sum_{\beta=1}^{D} x_{i}^{\beta}\left(J^{\prime}\right)_{i k}^{\beta \alpha}=\sum_{i=1}^{2 r-n} \sum_{\beta=1}^{D} x_{i}^{\beta} Y_{i k}^{\beta \alpha}-x_{k-(n-r)}^{\alpha},
$$

and, as the matrix $C$ above is orthogonal, the linear transformation which relates the new $\left\{y_{k}^{\prime}\right\}$ to the old $\left\{y_{k}\right\}$ has Jacobian equal to 1 . Defining

$$
x_{k-(n-r)}^{\alpha}\left(x_{1}, \ldots, x_{2 r-n} ; Y\right)=\sum_{i=1}^{2 r-n} x_{i}^{\beta} Y_{i k}^{\beta \alpha}, \quad k=r+1, \ldots, n,
$$

and a transposal matrix $Y^{T}$ such that $\left(Y^{T}\right)_{k i}^{\alpha \beta}=Y_{i k}^{\beta \alpha}$, the Gibbs weight (47) can be rewritten as

$$
\begin{aligned}
\exp \left[-\mathcal{H}_{J}(x)\right] & =\exp \left[-\frac{1}{2 \sigma^{2}}\left(\sum_{i=1}^{2 r-n}\left|x_{i}\right|^{2}+\sum_{j=2 r-n+1}^{r}\left|x_{j}(x ; Y)\right|^{2}\right)\right] \\
& =\exp \left[-\frac{1}{2 \sigma^{2}} \sum_{i, j=1}^{2 r-n} x_{i}^{\alpha}\left(I+Y Y^{T}\right)_{i j}^{\alpha \beta} x_{j}^{\beta}\right]
\end{aligned}
$$

which is of the form of the "simple" Gaussian model, equation (40), with $2 r-n$ variables, $n-r$ constraints, and all the $\sigma_{i}$ and the $\tau_{k}$ equal to $\sigma$, and the measure of integration $\mathrm{d} x$ stands for $\mathrm{d}^{D} x_{1} \cdots \mathrm{d}^{D} x_{2 r-n}$. So, with a slight abuse of notations, we can write the partition function of this model as

$$
Z_{\text {Gauss }}(Y, \sigma)=\int \mathrm{d} x \exp \left(-\frac{1}{2 \sigma^{2}} x^{\dagger}\left(I_{r}+Y Y^{T}\right) x\right),
$$

We could apply the duality to this formulation of the problem, using the general result of equation (43), obtaining

$$
Z_{\text {Gauss }}(Y, \sigma)=\frac{1}{(2 \pi)^{n-r}}\left(\frac{2 \pi}{\sigma}\right)^{\frac{n}{2}} Z_{\text {Gauss }}\left(-Y^{T}, \sigma^{-1}\right) .
$$

We could also apply the general duality directly to the original formulation. In direct formulation we have $r$ variables $x_{1}, \ldots, x_{r}$, with one-body functions $f_{i}\left(x_{i}\right)=$ $e^{-x_{i}^{2} /\left(2 \sigma^{2}\right)}$, and $(n-r)$ interactions, encoded in the delta functions $g_{k}\left(y_{k}\right)=\delta\left(y_{k}, 0\right)$. So that the matroid $B$ has the form $B=\left(I_{r}, J\right)$.

Accordingly to the general theory, the dual problem has $(n-r)$ variables $\eta_{1}, \ldots, \eta_{n-r}$, with constant one-body functions $\widehat{g}_{k}\left(\eta_{k}\right)=\widehat{\delta}\left(\eta_{k}\right)=1 /(2 \pi)$, and $r$ interactions given by

$$
\widehat{f}_{i}\left(\xi_{i}\right)=\sqrt{\frac{2 \pi}{\sigma}} \exp \left(-\frac{\sigma^{2} \xi_{i}^{2}}{2}\right) ; \quad \xi_{i}^{\beta}=\sum_{k=r+1}^{n} \sum_{\alpha=1}^{D} \eta_{k}^{\alpha}\left(-J^{T}\right)_{k i}^{\alpha \beta} .
$$

The dual matroid is given by $\widehat{B}=\left(-J^{T}, I_{n-r}\right)$, and the definition of the transposal matrix is $\left(-J^{T}\right)_{k i}^{\alpha \beta}=-J_{i k}^{\beta \alpha}$. At this point, we can remove the identity block from the matroid, as the functions associated to its columns are trivial constants. Then we can 
diagonalize the matrix $-J^{T}$ using the "transposal" of the procedure described above for $J$ (that is, moves on rows and columns are replaced by the correspondent moves on columns and rows), and obtain again the partition function on the right side of (53).

We remark that, in this case in which the entries of the matroid $B$ are non-trivial homomorphisms, we can check directly that, accordingly with the general recipe of section [5] the entries $\widehat{B}_{k i}$ of the dual matroid are related to the homomorphismtransposal of the entries $B_{i k}$ (that is, the internal indices $\alpha$ and $\beta$ are exchanged). Moreover, in agreement with the general description of section 7 we see how the resolution of delta constraints and the removal of constant factors are dual procedures.

\section{A generalized Potts-like model}

In this section we study the effects of the duality on a particular model which slightly generalizes the one we introduced in 4]. We deal with $r$ variables $x_{i} \in\{0, \ldots, q-1\}$ and a general hamiltonian with $(n-r)$ Potts-like interaction terms provided by a matrix $J$

$$
-\mathcal{H}\left(x ; J,\left\{K_{k}\right\},\left\{h_{i}\right\}\right)=\sum_{k=1}^{n-r} K_{k} \delta\left(\sum_{i} x_{i} J_{i k}\right)+\sum_{i=1}^{r} h_{i} \delta\left(x_{i}\right)
$$

where the sums and the delta functions are intended on $\mathbb{Z}_{q}$, that is $x \equiv x+n q$. The weights of the terms in the Hamiltonian are more conveniently expressed in terms of a vector $v \in \mathbb{R}^{n}$, defined as

$$
\begin{aligned}
v_{i}(x) & =e^{h_{i}}-1 & & \text { for } \quad i=1, \ldots, r ; \\
v_{r+k}(x) & =e^{K_{k}}-1 & & \text { for } \quad k=1, \ldots, n-r ;
\end{aligned}
$$

and of the functions

$$
f_{j}(x)=1+v_{j} \delta(x) .
$$

If we introduce the matroid $B=\left(I_{r} \mid J\right)$, we can reconduct the problem in the general form (15):

$$
\exp \left(-\mathcal{H}_{\mathbb{Z}_{q}, B,\left\{f_{j}\right\}}(x)\right)=\prod_{j=1}^{n}\left[1+v_{j} \delta\left((x B)_{j}\right)\right]
$$

We will introduce a notation ad hoc also for the partition function of this system:

$$
Z_{\text {Potts }}\left(J ;\left\{v_{j}\right\}\right) \equiv Z_{\mathbb{Z}_{q}, B,\left\{f_{j}\right\}},
$$

where the expressions (55) above for the $\left\{f_{j}\right\}$ are understood.

From the form of the Fourier transform of $f(x)=1+v \delta(x)$ :

$$
\widehat{f}(\xi)=\frac{v}{q}\left(1+\frac{q}{v} \delta(\xi)\right)
$$

and with the convention of Table 1 for the measure $\int_{x}$ in the case $X=\mathbb{Z}_{q}$, we have the relation between the partition functions

$$
Z_{\mathrm{Potts}}\left(-J^{T} ;\left\{q / v_{j}\right\}\right)=\frac{\prod_{i=1}^{r} v_{i}}{\prod_{k=1}^{n-r} q / v_{r+k}} Z_{\mathrm{Potts}}\left(J ;\left\{v_{j}\right\}\right) .
$$


Let us specialize the general duality transformation we have obtained to some simple cases, related to the model that we introduced in Ref. 4. First of all, we will consider the case in which the magnetic field is constant $\left(v_{j}=v\right.$ for all $\left.j=1, \ldots, r\right)$ and all the couplings are equals $\left(v_{j}=w\right.$ for all $\left.j=r+1, \ldots, n\right)$. The equation (59) in this case gives

$$
Z_{\mathrm{Potts}}\left(-J^{T} ; q / w, q / v\right)=\frac{v^{r}}{(q / w)^{n-r}} Z_{\mathrm{Potts}}(J ; v, w) .
$$

We can give a simple mathematical interpretation to the limit $v \rightarrow 0, w \rightarrow \infty$ of the formula above. In the two dual models, the partition functions, properly regularized, just count the number of solutions of the linear equations $x J=0$ and $y\left(-J^{T}\right)=0$. In this limit case the duality relation states that the rank of the matrix $J$ is equal to the rank of $-J^{T}$, which is a trivial fact of elementary geometry. This model is exactly solvable for the ensemble of random matrices on a finite field, that is we know the exact probability distribution for the free energy and other physical quantities, and the duality relation plays indeed a role in the solution of the problem 4 .

A particular case of (60) is when only $v \rightarrow \infty$ (and then $w^{\prime} \rightarrow 0$ ) where again the partition functions should be properly regularized. The explicit expression is

$$
\sum_{x} \delta(J x)(1+w)^{-\sum_{i}\left(1-\delta\left(x_{i}\right)\right)}=\sum_{\eta} \prod_{k}\left[\frac{w}{q}+\delta\left(\left(-J^{T} y\right)_{k}\right)\right],
$$

therefore, the duality relates a sum, restricted to the set of solutions of a linear system $J x=0$, where the difficulty of the problem is contained on the external field contribution, to a sum, with no external field, on all the states of the spectrum, where the difficulty is contained in the non-trivial correlations between the degeneracy of the energy levels. A quantitative study of this system will be done in a forthcoming paper [9].

We can use the general strategy of section 8 to investigate some sufficient conditions on the measure $\mu_{0}(J)$ for equation (60) to relate the partition functions of the same model at different values of the parameters $v$ and $w$.

As the weight functions involved are of the form "delta + constant", with no disorder parameters, the subgroup of automorphisms $A \subseteq \operatorname{Aut}\left(\mathbb{Z}_{q}\right)$ such that for a $a \in A$ we have $f(a(x))=f(x)$ is isomorphic to the classical group $\mathbb{Z}_{q}^{*}$. $\|$ That is, if $a$ is an integer coprime with $q$, the corresponding automorphism on $\mathbb{Z}_{q}$ acts as $a(x)=a x$ $\bmod q$.

The group of invariances obtained is sufficiently large that, following the strategy described in section 8 the self-duality condition can be verified for almost every reasonable ensemble of matrices. For example, this is the case of random matrices, of sparse matrices, both with non-zero elements chosen randomly among $\{1, \ldots, q-1\}$ or all equal to 1, and also of matrices with non-trivial correlations between the entries, if the correlations are symmetric under the exchanging of rows and columns, like the ensemble of incidence matrices of random bipartite graphs with vertices of fixed connectivity. An exception is the standard ensemble for the study of XOR-SAT problems [10, which is essentially a particular case of our model, but the matrices $J$ are constrained to have a fixed number of non-zero entries per row, and do not have any constraint on the columns.

$\|$ The group $\mathbb{Z}_{q}^{*}$ is defined as the integers in $\{1, \ldots, q-1\}$ which are coprime with $q$; the group operation is the product, and the standard modulo $q$ identification is understood. 
We note that, in the case of self-dual measure on the ensemble of matrices $J$, and $v=w$, the self-dual coupling occurs for $v=\sqrt{q}$, which is indeed the critical point also for the ordinary Potts model on a geometrically self-dual planar graph. We will show in the next section how to recover explicitly the self-duality in this case, in which other "geometrical" manipulations are required.

\section{Potts model on graphs and on planar graphs}

The model discussed in the previous section is a generalization of ordinary Potts model. In this section we will show how our duality relation can be reconducted to the common duality of Potts model on planar graphs [11].

Consider a connected graph $\Lambda$, at this stage not necessarily planar, with sites $s \in \mathcal{S}$, bonds $b \in \mathcal{B}$ and elementary cycles (or loops) $l \in \mathcal{L}$. Say that the number of sites, bonds and loops are respectively $S, B$ and $L$. If we consider the graph as embedded on an orientable surface, the set $\mathcal{L}$ of the loops could be chosen to be the set of the plaquettes ("local" loops), plus the set of the generators of the first homology group, that is, loops which turn around its handles. We remark that, at this point, this division is not necessary.

We will give an arbitrary orientation to the bonds and to the loops. That is, for each bond $b, s_{\text {in }}(b)$ and $s_{\text {out }}(b)$ will be respectively the first and second extremum of the bond. Furthermore, each loop $l$ will be associated with a $B$-dimensional vector $J_{b}(l)$, such that, having arbitrarily chosen an orientation for the loop, $J_{b}(l)$ is the number of times that the loop $l$ is occupied by the bond $b$ in the same orientation, minus the times it is occupied by $b$ in opposite orientation.

The Potts Model on the graph $\Lambda$ is defined by the set of variables $\sigma_{s} \in$ $\{0, \ldots, q-1\}$ on the sites, and a set of interactions with coupling constants $K_{b}$ on the bonds. I The Hamiltonian is

$$
-\mathcal{H}_{\substack{\text { Potts } \\ \text { Graph }}}\left(\sigma ; \Lambda,\left\{K_{b}\right\}\right)=\sum_{b \in \Lambda} K_{b} \delta\left(\sigma_{s_{\text {in }}(b)}-\sigma_{s_{\text {out }}(b)}\right) .
$$

If we introduce the functions

$$
\begin{aligned}
& f_{s}(x)=1 \\
& f_{b}(x)=1+v_{b} \delta(x) ; \quad v_{b}=e^{K_{b}}-1 ;
\end{aligned}
$$

and the matroid $B=\left(I_{S} \mid J_{s b}\right)$, with $J$ a $S \times B$ matrix defined as

$$
J_{s b}= \begin{cases}1 & s=s_{\text {in }}(b) \\ -1 & s=s_{\text {out }}(b) \\ 0 & \text { e.w. }\end{cases}
$$

the partition function

$$
Z_{\mathbb{Z}_{q}, B,\left\{f_{s}, f_{b}\right\}}=\sum_{\sigma} \prod_{s} f_{s}\left(\sigma_{s}\right) \prod_{b} f_{b}\left(\sum_{s} \sigma_{s} J_{s b}^{(1)}\right)
$$

I We introduce an additive group structure $\mathbb{Z}_{q}$ for the $q$ "colours" of the model, although ordinary Potts model Hamiltonian has a $\mathfrak{S}_{q}$ total permutational symmetry between the colours. We stress again the important fact that in general the symmetry group of the model is not related to the group introduced to perform the Fourier transform. 
is in the general form (16).

Now we remove the useless identity functions $f_{s}(x)$, and make the change of variables

$$
x_{b}(\sigma)=\sigma_{s_{\text {in }}(b)}-\sigma_{s_{\text {out }}(b)} .
$$

The weight of each configuration $\sigma$ is now just $\prod_{b} f_{b}\left(x_{b}(\sigma)\right)$. Next, we want to perform the sum of the partition function on the new variables $x$, instead that on the old $\sigma$. To this aim, we need to know how many configurations $\sigma$ correspond to a certain configuration $x$. The answer is simple: they are exactly $q$ if the circuitation of $x$ on each loop is zero (that is, $\sum_{b} J_{b}(l) x_{b} \equiv 0 \bmod q$ ), ${ }^{+}$and zero if the circuitation is non-zero for some loop. So we have

$$
\sum_{\sigma} \longrightarrow q \sum_{x} \prod_{l \in \mathcal{L}} \delta\left(\sum_{b} J_{b}(l) x_{b}\right),
$$

and, with the definitions

$$
\begin{aligned}
f_{b}(x) & =1+v_{b} \delta(x) ; & f_{l}(x) & =\delta(x) ; \\
J_{b l} & =J_{b}(l) ; & B^{\prime} & =\left(I_{B} \mid J_{b l}\right) ;
\end{aligned}
$$

we have again a system in the general form (16). At this point we can perform the duality transformation. According to the general formula (17), and to the expressions for the Fourier transforms of $f_{b}(x)$ and $f_{l}(x)$, respectively

$$
\widehat{f}_{b}(\xi)=\frac{v_{b}}{q}\left(1+\frac{q}{v_{b}} \delta(\xi)\right) ; \quad \widehat{f_{l}}(\xi)=1 ; \quad \widehat{B^{\prime}}=\left(-J_{l b} \mid I_{L}\right)
$$

the result of the transformation is a system in the original general form (65). If the matrix $-J_{l b}$ is of the kind of $J_{s b}$, with exactly two non-zero entries per column, a +1 and $\mathrm{a}-1$, we can reconstruct a dual graphical version of the original Potts model. As shown in detail in the Appendix A this always happens when the original graph is planar. In a few words, when a graph is planar, every bond appears in exactly two loops (the plaquettes on the two sides). If we choose a uniform orientation for the loops (all clockwise or all counter-clockwise), it appears in the two loops with opposite sign. So, in this dual model, two neighbouring plaquettes of the original graph appear to interact with the dual coupling constant of the common bond $\left(v_{b}^{\prime}=q / v_{b}\right)$. If we want to reconstruct a graph such that the system has the variables on the sites, and the interactions on the bonds, the desired graph is exactly the dual graph of the original one, in the sense of cell-complexes duality.

The procedure of this section can be applied with minor modifications to obtain the $X Y$-model - Coulomb Gas relation [12, 13], provided that the $X Y$-model Hamiltonian is in the Villain form. Essentially, the only difference is that the couple of dual groups is $\left(\mathbb{Z}_{q}, \mathbb{Z}_{q}\right)$ for the Potts Model, and $(U(1), \mathbb{Z})$ for the $X Y$-model Coulomb Gas relation.

\section{The coupling with gauge fields}

In this section we describe the application of the procedure described in section 6 to a very well known physical situation. The abstract framework that we introduced should

+ More generally, for $\Lambda$ not connected, we have a factor $q$ for each connected component. 
now assume a more transparent meaning: that procedure mimics what happens if we introduce a new set of gauge fields, coupled with the terms of interaction, and then a new term of the Hamiltonian depending from the physical content of these new fields.

The paradigm of this procedure is the introduction of minimal electrodynamic interaction in a scalar continuous field theory, with lagrangian

$$
\mathcal{L}_{0}=\int \mathrm{d} \phi\left[V(\phi)+\partial_{\mu} \phi \partial^{\mu} \phi\right] .
$$

The derivative operator $\partial_{\mu}$ has to be modified into a covariant derivative operator $D_{\mu}$, defined by the action on the fields as $D_{\mu} \phi=\partial_{\mu} \phi-A_{\mu}$. Then we have to add the dynamics of the gauge field, $F_{\mu \nu} F^{\mu \nu}$, where the field tensor is defined as $F_{\mu \nu}=\partial_{\mu} A_{\nu}-\partial_{\nu} A_{\mu}$. The new lagrangian will be

$$
\mathcal{L}_{\mathrm{QED}}=\int \mathrm{d} \phi \mathrm{d} A_{\mu}\left[V(\phi)+D_{\mu} \phi D^{\mu} \phi+\frac{1}{4 e^{2}} F_{\mu \nu} F^{\mu \nu}\right] .
$$

If we consider the statistical mechanic model whose Hamiltonian derives from the lattice discretized version of this model (as it is done, for example, in 14]), we replace partial derivatives $\partial_{\mu} \phi(x)$ with finite difference operators $(\partial \phi)_{n ; \mu}=\phi_{n+\widehat{\mu}}-\phi_{n}$, so that, as usual, the interactions lie on the bonds of the lattice. The linear combinations $(\partial \phi)_{n ; \mu}$ are the arguments of the interaction terms in the Gibbs factor.

The discretized gauge fields $A_{n ; \mu}$ couple to the terms of interactions, in such a way that the finite difference of the fields, $(\partial \phi)_{n ; \mu}$, are modified into the covariant derivative $(D \phi)_{n ; \mu}=(\partial \phi)_{n ; \mu}-A_{n ; \mu}$.

The discretized field tensor $F_{n ; \mu \nu}=A_{n ; \mu}+A_{n+\widehat{\mu} ; \nu}-A_{n+\widehat{\nu} ; \mu}-A_{n ; \nu}$ takes the circuitation of the vector field $A_{n ; \mu}$ around each elementary plaquette $(n ; \mu \nu)$ of the lattice, and thus is a linear combination of the entries $A_{n ; \mu}$.

The current quadrivector is given by the divergence of the electromagnetic tensor, $j_{n, \mu}=\sum_{\nu}\left(F_{n, \mu \nu}-F_{n-\nu, \mu \nu}\right)$, and its divergence, which is automatically zero, is given by $(\nabla \cdot j)_{n}=\sum_{\mu}\left(j_{n, \mu}-j_{n-\mu, \mu}\right)$.

We can generalize this frame to an abstraction of the geometric structure of a lattice, in which we have a set of sites $\{s\}$, a set of bonds $\{b\}$, a set of plaquettes $\{p\}$ and a set of dual sites $\left\{s^{\prime}\right\}$. In the previous case we would have for the sites $\{s\}=\{n\}$, for the bonds $\{b\}=\{(n, \mu)\}$, for the plaquettes $\{p\}=\{(n, \mu \nu)\}$, and for the dual sites $\left\{s^{\prime}\right\}=\{(n, \mu \nu \rho)\}$.

The action of gradient and divergence operators is now performed via some adjacence matrices. In particular we will introduce three matrices $J_{s b}, J_{b p}$ and $J_{p s^{\prime}}$, such that, given the two sets respectively of scalar fields $\phi_{s}$ and of gauge fields $A_{b}$, we have

$$
(D \phi)_{b}=\phi_{s} J_{s b}-A_{b} ; \quad F_{p}=A_{b} J_{b p} ; \quad(\nabla \cdot j)_{s^{\prime}}=F_{p} J_{p s^{\prime}} .
$$

We will be interested in the theory with Gibbs weight

$$
\exp \left(-\mathcal{H}_{\mathrm{QED}}(\phi, A)\right)=\prod_{s} f_{s}\left(\phi_{s}\right) \prod_{b} f_{b}\left((D \phi)_{b}\right) \prod_{p} \widehat{g}_{p}\left(F_{p}\right) \prod_{s^{\prime}} \widehat{g}_{s^{\prime}}\left((\nabla \cdot j)_{s^{\prime}}\right),
$$

with the four set of functions given by

$$
\begin{array}{llrl}
f_{s}(x) & =1 ; & f_{b}(x) & =\exp \left(-\frac{1}{2} x^{2}\right) ; \\
\widehat{g}_{p}(x) & =\exp \left(-\frac{1}{4 e^{2}} x^{2}\right) ; & \widehat{g}_{s^{\prime}}(x) & =\delta(x) .
\end{array}
$$




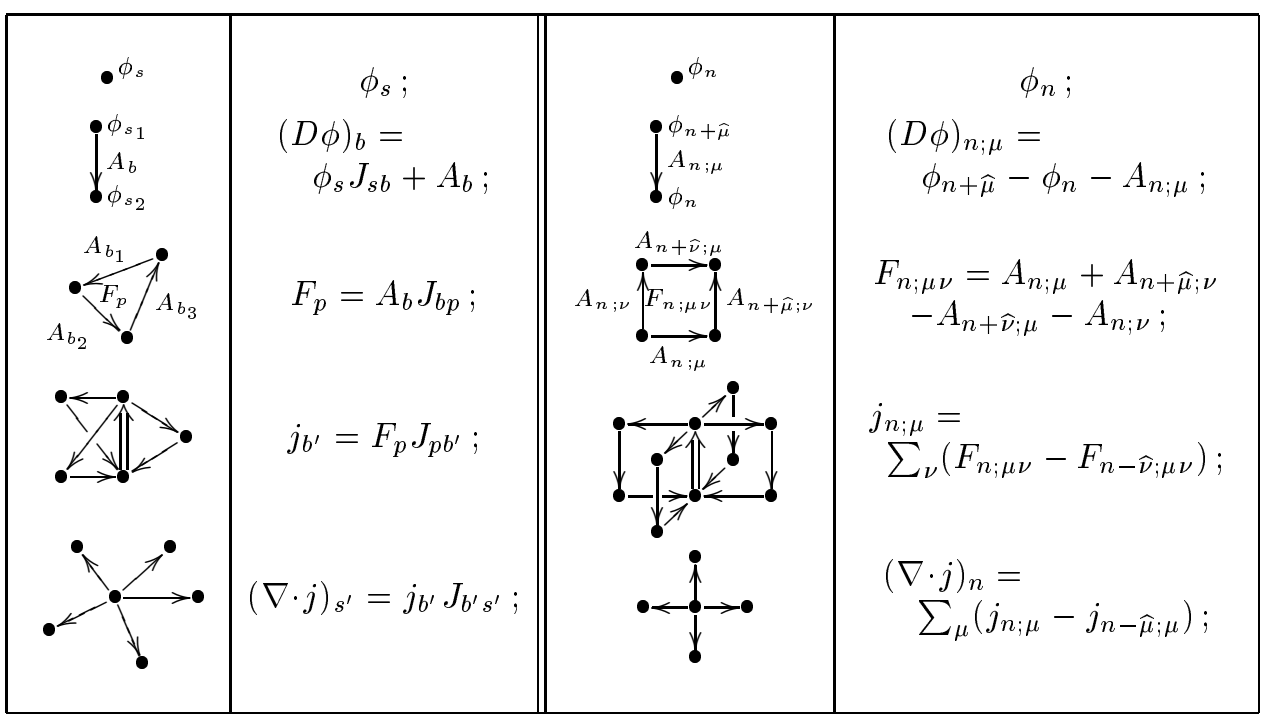

Figure 1. A table which collects the most important quantities in discretized versions of QED, for regular lattices (right), and for arbitrary graphs (left).

In our general frame of section 4 this model corresponds to the matroid

$$
B=\left(\begin{array}{cccc}
I & -J_{s b} & & \\
& I & J_{b p} & J_{b p} J_{p s^{\prime}}
\end{array}\right)
$$

which is in the general form described in section [6] Following the procedure described therein, we obtain for the dual matroid

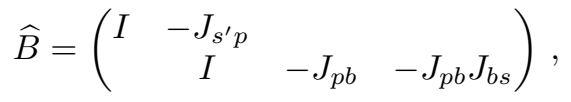

in which sites are exchanged with dual sites, bonds are exchanged with plaquettes, and the relative orientation of bonds and plaquettes is reversed (in the geometrical duality for three-dimensional lattices, this corresponds to the fact that the orientations of bonds and dual bonds change in such a way that the vector product changes parity).

Introducing the ad hoc notation for the partition function

$$
Z_{\mathrm{QED}}(e, B)=\int \mathrm{d} \phi_{s} \mathrm{~d} A_{b} \exp \left(-\frac{1}{2} \sum_{b}(D \phi)_{b}^{2}-\frac{1}{4 e^{2}} \sum_{p} F_{p}^{2}\right) \prod_{s^{\prime}} \delta\left((\nabla \cdot j)_{s^{\prime}}\right),
$$

with the proper normalizations of the integrals, the duality relation states that

$$
Z_{\mathrm{QED}}(e, B)=\frac{(2 \pi)^{\frac{r}{2}}}{\left(4 \pi e^{2}\right)^{\frac{n-r}{2}}} Z_{\mathrm{QED}}\left(\frac{1}{2 e}, \widehat{B}\right)
$$

where, as always, $r$ is the rank of $B$, and in our case is equal to the number of sites plus the number of bonds, and $(n-r)$ is the rank of $\widehat{B}$, and in our case is equal to the number of dual sites plus the number of plaquettes. Again we see how duality exchanges a regime of high coupling with a regime of low coupling. 
A concrete example could be the one of a cubic three-dimensional lattice, with the ordinary notions of sites, bonds and plaquettes, and the dual sites being the elementary cubic cells of the lattice. The geometric duality applied to the lattice naturally exchanges sites with dual sites and bonds with plaquettes. In this case, the presence of the delta function for the terms of current divergence is redundant, as the constraint is automatically satisfied.

Variants in the spirit of disordered systems could involve a measure $\mu(B)$ over an ensemble of random tessellations of the three-dimensional space, or, more generally, over an ensemble in which adjacency matrices are not directly derived from a geometrical structure, and the condition $\nabla \cdot j=0$ really needs to be enforced via a delta function in the Gibbs weight.

In the case in which, following the recipe of section 8 we have a self-dual measure $\mu^{*}(B)$, the fixed point of the involution for the electric charge $e$ is at the value $2 e^{2}=1$.

\section{Conclusions}

The notion of duality 'a la Kramers and Wannier, after its original introduction in 1941, has been applied to a wide range of periodic finite-dimensional systems of statistical mechanics. On the contrary, small work has been done in the direction of a systematic description of duality for a class of models sufficiently large to include, for example, mean-field disordered systems.

Our idea in this paper is that, in the Kramers and Wannier formulation, a sort of two-step procedure is implicit: an algebraic duality, which exchanges variables and interactions, and performs a Fourier transform on the terms of the Gibbs weight, and a geometric duality, which, exploiting the geometrical structure of the lattice, allows for a reinterpretation of the dual system in a formulation which, in some contexts, is suitable for a direct comparison with the original system.

In the more wide context of disordered systems the second step is in general impossible. Nonetheless, in many cases the first step is already suitable for a fruitful comparison of the two systems related by duality.

The general recipe is the following: given some variables and some interactions, valued on some abelian group $G$, the Gibbs weight depends from two ingredients:

- two sets of functions, weighting respectively the original variables, and the auxiliary variables corresponding to some linear combinations of the original ones (the interactions);

- the specific pattern of linear combinations of the variables, encoded in a matrix $J$ of group-homomorphisms.

The dual system exchanges variable-functions and interaction-functions with the Fourier anti-transform and transform of interaction-functions and variable-functions, and the new pattern is given by the matrix $-J^{T}$ of transposed homomorphisms.

We give some concrete applications for specific simple models: a random Gaussian model, a random Potts-like model, a random variant of discrete scalar QED... For each of these systems, a short discussion over the duality relation is proposed, and a hint over self-duality conditions is suggested. Actually, in the cases of interest, this discussion is a simple specialization of a general discussion done in section 8 , and analogous to the one already present in [4, where the duality, in its first application, was called variable-clause duality. 
An unexpected connection with Matroid Theory has emerged in the analysis of the subject. The a posteriori justification for this fact is that the pattern of interaction which is involved in the definition of the Gibbs weight is intrinsic with respect to a choice of basis for the space of configurations. Matroid Theory, in simple words, describes mathematical objects which convey basis-intrinsic information on matrixlike objects, like the pattern of linear dependence between the columns of a matrix. Hence, it is small surprise if the matroid related to the dual system is the matroid-dual of the matroid related to the original system.

In a forthcoming paper we would discuss a further generalization of this framework, in which the crucial concepts of Fourier transform and of grouphomomorphism for abelian groups are generalized to different integral transform and natural notions of homomorphism, applied to mathematical structures different from abelian groups. A first case is the one in which, for Partially Ordered Sets, the natural notion of transform is the Möbius transform, and the natural notion of homomorphisms is related to the theory of Galois connections [15].

\section{Appendix A. Duality for graphical matroids and planar graphs}

An important subset of regular matroids $\ddagger$ is the set of graphical matroids. Many results exist on the subject (cfr., for example, [8], chapt. 5), derived in the spirit of generality characteristic of Matroid Theory. In this appendix we will show some constructive procedures which apply to particular classes of graphical matroids, so in this sense they are less general, but more clear restatements of known facts. Hopefully, they will be useful to understand how to interpret the classical formulations of dualities for SM models on planar lattices inside our general setting of the duality.

A precise terminology will be introduced in the body of the section. Here we resume the main results:

Theorem 1 The "site-bond" matroid $J_{s b}$ and the "loop-bond" matroid $J_{l b}$ of a graph $\mathcal{G}$ are dual matroids.

Theorem 2 The "site-bond" matrix $J^{\prime}{ }_{s b}$ of a planar graph $\mathcal{G}$ is equal to the "loopbond" matrix $J^{\prime}$ lb of the corresponding geometrical dual graph $\mathcal{G}^{*}$, and conversely the "loop-bond" matrix of $\mathcal{G}$ is equal to the "site-bond" matrix of $\mathcal{G}^{*}$.

\section{Appendix A.1. Matroid duality for graphical matroids}

Consider a graph $\mathcal{G}$, with vertices (or sites) $s \in V$ and edges (or bonds) $b \in E$. We give an arbitrary orientation to the bonds. That is, for each bond $b, s_{\text {in }}(b)$ and $s_{\text {out }}(b)$ will be respectively the first and second extremum of the bond.

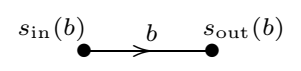

Define a 0 -form $\varphi$ as a function from $V$ to a field $\mathbb{F}$, and a 1 -form $\psi$ as a function from $E$ to $\mathbb{F}$. The operators $\partial$ and $\partial^{*}$ transform 0 -forms in 1 -forms and vice versa with the following definition:

$$
\begin{aligned}
\partial \varphi(b) & =\varphi\left(s_{\text {in }}(b)\right)-\varphi\left(s_{\text {out }}(b)\right) ; \\
\partial^{*} \psi(s) & =\sum_{b} \psi(b)\left(\delta_{s, s_{\text {in }}(b)}-\delta_{s, s_{\text {out }}(b)}\right) .
\end{aligned}
$$

$\ddagger$ That is, matroids which are representable over every field. 
Intuitively, they correspond to the discretization of the gradient operator on scalar fields and to the divergence operator on vector fields, generalized to arbitrary lattices.

The sites are implicitly a particular case of 0 -form, $\varphi_{(s)}\left(s^{\prime}\right)=\delta_{s, s^{\prime}}$. The set of 0 -forms is a vector space over $\mathbb{F}$, of dimension $|V|$. It is naturally decomposed in two vector spaces, $\mathcal{S}$ and $\mathcal{C}$, where 0 -forms in $\mathcal{C}$ are constant on each of the $C$ connected components of $\mathcal{G}$ (so $\operatorname{dim}(\mathcal{C})=C$ ), and 0 -forms in $\mathcal{S}$ are obtained by quotient (so $\operatorname{dim}(\mathcal{S})=S=|V|-C)$. Projection on $\mathcal{S}$ is obtained via the operator $\frac{1}{2} \partial^{*} \partial$.

Analogously, the set of 1 -forms is a vector space $\mathcal{B}$ over $\mathbb{F}$, of dimension $B=|E|$. The bonds are a particular case of generic 1-forms, where it is understood $\psi_{(b)}\left(b^{\prime}\right)=$ $\delta_{b, b^{\prime}}$, and are a basis for the space $\mathcal{B}$. We will define the loops as the 1 -forms $\psi$ such that $\partial^{*} \psi=0$. Elementary plaquettes are particular cases of loops: if $\partial p$ is the border of an oriented plaquette $p, \psi_{(p)}(b)$ is the characteristic function on $\partial p$. The set of loops is a vector space $\mathcal{L}$ over $\mathbb{F}$, of dimension $L$.

Note that $S+L=B$ as a consequence of Euler formula. More deeply, the vector space $\mathcal{B}$ is decomposed into $\partial \mathcal{S}+\mathcal{L}$, that is, a generic 1 -form $\psi$ is uniquely decomposed into $\psi=\partial \varphi+\chi$, with $\varphi \in \mathcal{S}$ and $\chi \in \mathcal{L}$ (Hodge decomposition).

The patterns of linear dependence of the set of bonds projected onto the vector spaces $\partial \mathcal{S}$ and $\mathcal{L}$ are matroids. They will be called respectively the site-bond and the loop-bond matroids. Given a choice of basis for $\mathcal{S}$ and $\mathcal{L}$, we will denote the corresponding vectorial representations as $J_{s b}$ and $J_{l b}$. That is, for a set of 0 -forms $\left\{\varphi_{s}\right\}$ chosen as a basis for $\mathcal{S}$, the entries of $J_{s b}$ will be

$$
J_{s b}=\partial \varphi_{s}(b),
$$

and for a set of 1 -forms $\left\{\psi_{l}\right\}$ chosen as a basis for $\mathcal{L}$, the entries of $J_{l b}$ will be

$$
J_{l b}=\psi_{l}(b) .
$$

We will give a particularly skill choice of basis, for which the two matroids are both in standard vectorial form, and manifestly dual.

Choose a "ground" site $s_{0} \in V$, and a spanning tree $\mathcal{T} \subseteq \mathcal{G}$. Change the orientation of the bonds such that for each site $s \neq s_{0}$ there is exactly one outgoing bond in $E(\mathcal{T})$. Label this bond $b_{s}$. Say that $s \succ s^{\prime}$ iff an oriented path $\gamma_{s^{\prime} \rightarrow s}$ in $\mathcal{T}$ exists. Choose a basis for $\mathcal{S}$ as

$$
\varphi_{s}\left(s^{\prime}\right)= \begin{cases}+1 & s \succ s^{\prime} \\ 0 & \text { o.w. }\end{cases}
$$

So we have

$$
\partial \varphi_{s}\left(b_{s^{\prime}}\right)=\delta_{s, s^{\prime}} ; \quad \partial \varphi_{s}\left(b_{l}\right)= \begin{cases}+1 & s \succ s_{\text {in }}\left(b_{l}\right), s \nsucc s_{\text {out }}\left(b_{l}\right) \\ -1 & s \succ s_{\text {out }}\left(b_{l}\right), s \nsucc s_{\text {in }}\left(b_{l}\right) \\ 0 & \text { o.w. }\end{cases}
$$

We will choose a basis for $\mathcal{L}$ such that for each bond $b \in E \backslash E(\mathcal{T})$ there is exactly one 1-form $\psi_{l}$ such that $\psi_{l}(b)$ is non-zero. So we can label the edges in $E \backslash E(\mathcal{T})$ as $b_{l}$. More precisely, the 1-form $\psi_{l}$ corresponds to the only self-avoiding closed path on $\mathcal{T} \cup\left\{b_{l}\right\}$, oriented according to $b_{l}$. Analytically this can be expressed as

$$
\psi_{l}\left(b_{l^{\prime}}\right)=\delta_{l, l^{\prime}} ; \quad \psi_{l}\left(b_{s}\right)= \begin{cases}-1 & s \succ s_{\text {in }}\left(b_{l}\right), s \nsucc s_{\text {out }}\left(b_{l}\right) \\ +1 & s \succ s_{\text {out }}\left(b_{l}\right), s \nsucc s_{\text {in }}\left(b_{l}\right) \\ 0 & \text { o.w. }\end{cases}
$$


It is immediate to check that $J_{s b}$ and $J_{l b}$ are in standard vectorial form, respectively with the identity block on the left and on the right. From the observation that $\partial \varphi_{s}\left(b_{l}\right)=-\psi_{l}\left(b_{s}\right)$ we obtain that they are dual in matroid sense:

$$
J_{s b}=\left(I, Y_{s l}\right) ; \quad J_{l b}=\left(-Y_{l s}^{T}, I\right) .
$$

As we have found an explicit choice of basis for which the standard form of the sitebond matroid and of the loop-bond matroid are dual, we have implicitly proved that the matroids are dual, which is the claim of Theorem 1.

\section{Appendix A.2. Graphical duality for planar graphs}

In the previous section we have seen how the "abstract" site-bond and the loop-bond matroids for a given graph are dual in the sense of matroid duality. This is true in general, as the matroid definition is intrinsic, and specifically we have verified it in a particular choice of basis, for which both the matroids are in standard form.

In this section we show the result of Theorem 2, that the site-bond and the loopbond incidence matrices for a connected planar graph and for its graphical dual are exchanged by the graphical duality. This result relies on a particular choice for the loops as the elementary plaquettes of the lattice, which is the more natural for the case of planar graphs, but in this sense is more accidental.

A graph $\mathcal{G}$ is said to be planar when it is embeddable on a genus-0 surface with no crossings between the bonds. Consider the case in which $\mathcal{G}$ is connected. The classical Euler relation for this kind of graphs claims that $V+F=B+2$, where $V$ is the number of sites, $B$ is the number of bonds and $F$ is the number of faces.

One can immediately define the site-bond and loop-bond incidence matrices, $A_{s b}$ and $A_{l b}$, for non-oriented bonds and loops. Just state that $A_{s b}$ is 1 if the site $s$ is an extremum of the bond $b$ and 0 elsewhere, and $A_{l b}$ is 1 if the bond $b$ is a side of the face $l$ and 0 elsewhere.

The graphical dual $\mathcal{G}^{*}$ is defined by the following procedure. Replace each plaquette $l$ by a dual vertex $s^{*}$ (white bullets in Fig. 10), each bond $b$ by a dual (dashed) bond $b^{*}$, which crosses it and connects the dual vertices of the two neighbouring plaquettes, and each site $s$ with the dual plaquette $l^{*}$ delimited by the dual bonds of the bonds outgoing from the site. It is clear that

$$
A_{s^{*} b^{*}}\left(\mathcal{G}^{*}\right)=A_{l b}(\mathcal{G}) ; \quad A_{l^{*} b^{*}}\left(\mathcal{G}^{*}\right)=A_{s b}(\mathcal{G}) .
$$

If we put an orientation on the bonds and a direction of rotation on the faces, the matrices $A_{s b}$ and $A_{l b}$ will be promoted to some matrices $J_{s b}^{\prime}$ and $J_{l b}^{\prime}$, in which some minus signs appear. Again because of the planarity of the graph, we could give a recipe such that the relation A.10 above is promoted to a relation

$$
J_{s^{*} b^{*}}^{\prime}\left(\mathcal{G}^{*}\right)=J_{l b}^{\prime}(\mathcal{G}) ; \quad J_{l^{*} b^{*}}^{\prime}\left(\mathcal{G}^{*}\right)=J_{s b}^{\prime}(\mathcal{G}) .
$$

The recipe is the following: choose the direction of rotation to be the same for all the faces, (for example clockwise), and opposite between faces and dual faces. Choose the orientation of the bonds arbitrarily. The orientation of the dual bonds must be such that the vector product between each bond and its dual bond has the same sign with respect to the normal versor outgoing from the surface.

Both the matrices $J_{s b}^{\prime}$ and $J_{l b}^{\prime}$ are not of maximal rank, and the only linear relation is given by the sum of all the rows with equal coefficients. This accounts for the offset 

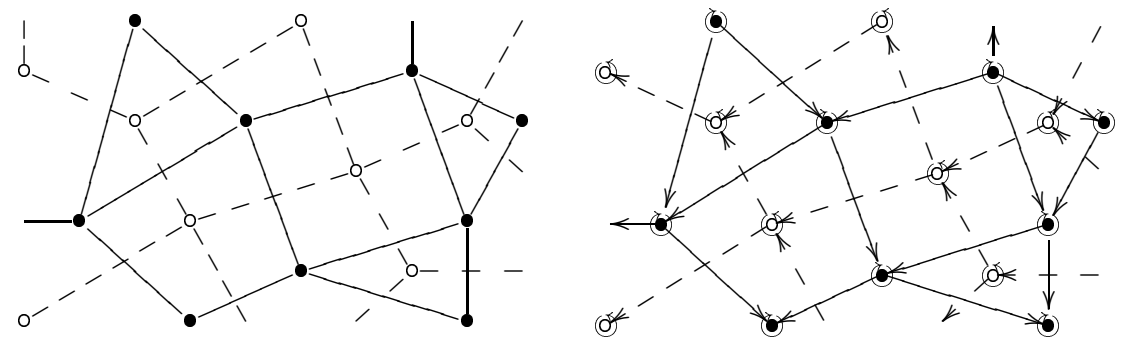

Figure A1. Graphical dual for a planar graph (left), and a consistent choice of orientations for the bonds and directions of rotations for the faces for the relation A.11 to hold (right).

of 2 in the Euler formula $V+F=B+2$, in fact the number of "independent sites" in the loop-bond matroid (0-forms up to an arbitrary constant) is $S=V-1$, and the number of independent loops is $L=F-1$, from which we recover the relation $S+L=B$.

\section{Acknowledgments}

We thank Alan Sokal for introducing us to the relevance of the theory of matroids for

Potts-like systems, and for valuable discussions in the reading of the manuscript.

\section{References}

[1] H. A. Kramers and G. H. Wannier, Phys. Rev. B 19 (1941), 3689-99

[2] K. Drühl and H. Wagner, Algebraic formulation of duality transformations for abelian lattice models, Ann. of Phys. 141, 225-253 (1982)

[3] R. Savit, Duality transformations for general abelian systems, Nucl. Phys. B 200, 233 (1982); R. Savit, Duality in field theory and statistical systems, Rev. Mod. Phys. 52, 453-487 (1980)

[4] S. Caracciolo and A. Sportiello, An exactly solvable random satisfiability problem, J. Phys. A 35, 7661-88 (2002), cond-mat/0206352

[5] A.A. Kirillov, A.D. Gvishiani, Theorems and Problems in Functional Analysis, Springer-Verlag, New York, 1982

[6] E. Hewitt and K.A. Ross, Abstract Harmonic Analysis Vol. 1, Series of Comprehensive Studies in Mathematics 115, Springer-Verlag, Berlin (1979)

[7] H. Whitney, On the abstract properties of linear dependence, Amer. J. Math. 57 (1935), 509533

[8] J. Oxley, Matroid Theory (Oxford University Press, Oxford, 1992).

[9] S. Caracciolo and A. Sportiello, Finite-temperature statistical mechanics of a random satisfiability problem, Preprint IFUM-750-FT.

[10] F. Ricci-Tersenghi, M. Weigt, and R. Zecchina, Simplest random K-satisfiability problem, Phys. Rev. E 63, 026702 (2001)

[11] R.B. Potts, Some generalized order-disorder transformations, Proc. Cambridge Philos. Soc. 48, 106-109 (1952)

[12] J. Villain, Theory of one-dimensional and two-dimensional magnets with an easy magnetization plane. 2. The planar, classical, two-dimensional magnet, J. Phys. (Paris) 36, 581 (1975)

[13] B. Nienhuis, Two-dimensional critical phenomena and the Coulomb Gas, Review in the series Phase Transitions and Critical Phenomena, Eds. C. Domb, M. Green and J.L. Lebowitz. Vol. 11, Academic Press, London (1987)

[14] M.E. Peskin, Mandelstam-'t Hooft duality in abelian Lattice Models, Ann. of Phys. 113, 122-152 (1978)

[15] S. Caracciolo and A. Sportiello, Duality for statistical mechanics models and Möbius transform on partially ordered sets, in preparation. 